\title{
Médiévales
}

Langues, Textes, Histoire

55 | automne 2008

Usages de la Bible

\section{Daniel et les visions politiques à l'époque carolingienne}

Daniel and Political Visions in the Carolingian Era

\section{Sumi Shimahara}

\section{(2) OpenEdition}

\section{Journals}

Édition électronique

URL : http://journals.openedition.org/medievales/5437

DOI : 10.4000/medievales.5437

ISSN : 1777-5892

Éditeur

Presses universitaires de Vincennes

\section{Édition imprimée}

Date de publication : 20 décembre 2008

Pagination : 19-32

ISBN : 978-2-84292-221-4

ISSN : 0751-2708

\section{Référence électronique}

Sumi Shimahara, "Daniel et les visions politiques à l'époque carolingienne », Médiévales [En ligne], 55 | automne 2008, mis en ligne le 20 mars 2011, consulté le 01 mai 2019. URL : http://

journals.openedition.org/medievales/5437; DOI : 10.4000/medievales.5437

Ce document a été généré automatiquement le 1 mai 2019.

Tous droits réservés 


\section{Daniel et les visions politiques à l'époque carolingienne}

Daniel and Political Visions in the Carolingian Era

\section{Sumi Shimahara}

1 Le livre de Daniel est célèbre pour ses épisodes hagiographiques, en particulier pour ses miracles ordaliques, tels que celui des enfants dans la fournaise (Dn 3) ou de Daniel dans la fosse aux lions (Dn 6, Dn 14), mais aussi pour le récit du jugement de Suzanne (Dn 13). Tous ces passages ont une forte charge eschatologique : ils font référence au Jugement dernier et à la résurrection finale. C'est la raison pour laquelle ils ont été abondamment utilisés dans la liturgie depuis l'époque patristique ${ }^{1}$. Mais le charisme du saint se manifeste surtout par sa capacité à interpréter des visions, ou à en recevoir l'explication extraordinaire par l'intermédiaire des anges. Dans le récit biblique, cette intelligence exceptionnelle des signes est mise au service des rois : Daniel les conseille, les avertit, les exhorte à écouter la parole de Dieu et à agir en conséquence. Si les prophètes vétérotestamentaires contestent souvent la légitimité de l'institution en place, Daniel est au contraire un homme de Dieu au service du pouvoir politique en place, même si c'est pour le mettre en garde contre ses déviances. Certaines de ses prophéties ont donc une dimension politique : le songe de la statue aux pieds d'argile mêlée de fer (Dn 2), celui de l'arbre coupé (Dn 4), la vision des quatre bêtes successives (Dn 8) ont été très tôt interprétés comme autant de récits relatifs à caducité du pouvoir des rois du monde.

Cela fait du livre une sorte de miroir des princes, tout au moins à certaines époques. La période carolingienne est de celles-là : temps d'une réforme qui s'appuie sur la Bible, d'une restauration chrétienne de l'Empire romain qui s'identifie à l'Église, le IXe siècle latin lit et relit le livre de Daniel. Entre le commentaire de Jérôme, composé à l'aube du v siècle, et celui de Pierre de Pise, grammairien à la cour de Charlemagne à la fin du VIII ${ }^{\mathrm{e}}$ siècle, on ne garde la trace d'aucun traité consacré au livre de Daniel en Occident. De la fin du viII ${ }^{\mathrm{e}}$ siècle jusqu'au $\mathrm{x}^{\mathrm{e}}$ siècle, cinq exégètes expliquent ce prophète : Pierre de Pise, l'auteur anonyme de la Glose de Saint-Gall, Raban Maur, Haymon d'Auxerre et un anonyme qui pourrait être Israël $\mathrm{Scot}^{2}$. Ce regain d'intérêt n'est pas spécifique à Daniel : 
les Carolingiens commentent abondamment la Bible puisqu'ils cherchent à réformer la société selon les prescriptions du Livre. Mais Daniel profite davantage de ce mouvement que le corpus des petits prophètes par exemple : ce dernier n'est expliqué que par trois auteurs, Haymon d'Auxerre, l'anonyme qui composa la Glose de Saint-Gall et Otfrid de Wissembourg 3 . Un autre indice montre l'intérêt porté à Daniel à l'époque carolingienne : ses récits servent de matrice à une vision rédigée au cours du $\mathrm{IX}^{\mathrm{e}}$ siècle.

En quoi ce prophète permet-il aux Carolingiens de comprendre la réalité politique de leur temps et d'agir sur elle? Nous présenterons trois éléments de réponse. Une des raisons du succès du livre réside certainement dans le thème de la succession des empires, qui a pu paraître d'actualité après l'éclatement de celui des Carolingiens en 843. De fait, seul le commentaire de Pierre de Pise est antérieur de manière certaine à cette date ${ }^{4}$. En outre, le discours politique est alors marqué par ce qu'Yves Congar a nommé le «moralisme carolingien ${ }^{5}$ » : le pouvoir est fortement personnalisé, sa pratique est évaluée à l'aune de critères moraux illustrés par des modèles bibliques. Les divers récits de Daniel fournissent plusieurs exemples de contre-modèles royaux, que le prophète tente de remettre dans le droit chemin. Tout ce qui a trait à la caducité du pouvoir terrestre semble avoir fait écho à la désagrégation de l'empire carolingien. Enfin le rôle du prophète, interprète des signes envoyés par Dieu et de ce fait conseiller du roi, a également rencontré une attente $\mathrm{au} \mathrm{IX}^{\mathrm{e}}$ siècle: les exégètes détiennent souvent cette fonction et se sont reconnus en Daniel.

Daniel est, indubitablement, un livre d'histoire: Fréculphe de Lisieux s'y réfère régulièrement, à propos de la prise de Jérusalem par Nabuchodonosor et de la déportation des juifs à Babylone, ou à propos de la chute de Balthasar et de la succession des empires ${ }^{6}$. Il est vrai qu'en ce dernier lieu, il suit les Antiquités judaïques de Flavius Josèphe, après avoir évoqué Orose : la tradition historique de recours à la Bible est déjà bien ancrée ${ }^{7}$. Fréculphe se fonde aussi sur Daniel pour évoquer la date de l'incarnation ou la destruction du Temple de Jérusalem par les Romains ${ }^{8}$. On sait que le thème de la succession des empires est lu de deux manières à l'époque carolingienne: Orose en offre une vision plutôt optimiste, tandis que Jérôme y lit une déchéance. Cette dernière lecture a davantage inspiré l'exégèse et les visions carolingiennes.

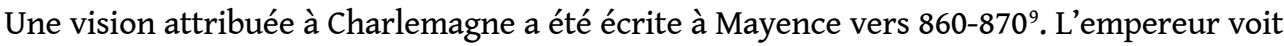
en songe quelqu'un lui offrir une épée, au nom de Dieu. L'inconnu lui enjoint d'être attentif à ce qui est inscrit sur la lame. Charlemagne, une fois seul, découvre en effet quatre mots (dans une langue énigmatique des songes) : raht, radoleiba, nasg, enti. Il se réveille et, après avoir prié, demande à ses conseillers l'explication de ce songe. Tous se taisent. Éginhard ose finalement prendre la parole : c'est au donateur inconnu de l'épée de révéler le sens. Charlemagne propose alors son interprétation: l'épée que Dieu lui donne symbolise l'aide divine qui a permis au roi de remporter bien des victoires militaires, et donc de léguer à ses descendants un État prospère (raht). Le règne de son successeur, en revanche, est marqué par le signe du manque (radoleiba) : ce roi ne saura pas faire fructifier l'héritage. Les enfants de ce dernier souverain seront pires encore: pour pallier le manque, ils seront âpres au gain, prêts à tous les vols (nasg), y compris à l'égard des biens ecclésiastiques. C'est ainsi qu'ils mèneront la dynastie à sa fin (enti). Selon celui qui met par écrit la vision, le récit aurait été rapporté par Éginhard à Raban. C'est grâce à ce dernier que le rédacteur affirme en connaître le contenu. L'auteur conclut son récit en remarquant que le temps écoulé a permis l'accomplissement des prédictions : les invasions et la pénurie ont marqué le règne de Louis; Pépin et Lothaire se sont 
attaqués aux biens ecclésiastiques de leurs royaumes. La sainte Église romaine, après avoir été dépouillée, puis humiliée, est désormais anéantie.

6 On reconnaît dans ce récit plusieurs épisodes de Daniel : le thème de la succession des empires s'inspire des chapitres 2 et 7 , soit respectivement de la vision de la statue aux pieds d'argile et de la vision des quatre bêtes; les mots étranges adressés au souverain rappellent l'épisode du festin de Balthasar (Dn 5), quand une main trace sur le mur des mots difficiles à comprendre mais qui annoncent l'avenir immédiat du roi. Tandis que dans le livre biblique, c'est le prophète qui, avec l'aide de Dieu, interprète la vision, dans le récit carolingien, c'est Charlemagne lui-même qui est inspiré. Cela revient à lui attribuer un rôle prestigieux et à souligner le déclin progressif de sa dynastie. Daniel est utilisé ici pour dénoncer une décadence. Tel est également le cas dans le récit biographique que Notker de Saint-Gall consacre à Charlemagne. Une fois l'Empire romain effondré, une nouvelle statue à tête d'or est édifiée. Cette tête est celle de Charlemagne, et les autres matériaux correspondent à ses descendants, qui fragilisent son héritage ${ }^{10}$.

7 Ces deux exemples montrent combien l'exégèse de Jérôme, qui voyait dans la succession des empires un déclin progressif, imprègne la pensée carolingienne. De fait, tous les exégètes reprennent alors l'interprétation hiéronymienne. En Dn 2, 31-35, à propos de la statue aux pieds d'argile mêlée de fer, Raban reproduit mot pour mot l'explication de Jérôme qui voyait dans les pieds, l'Empire romain, et dans leur matière fragile, l'accomplissement, à son époque, de la prophétie : «Mais ses pieds et ses doigts sont en partie de fer et en partie d'argile, ce que notre époque confirme très clairement: si, au commencement, rien n'était plus fort ni plus résistant que l'Empire romain, à la fin, il n'est rien de plus faible, puisque, dans les guerres civiles et aussi face à diverses nations, nous avons besoin d'autres peuples barbares ${ }^{11}$. »

8 Le changement de contexte n'amoindrit pas la portée des mots de Jérôme, bien au contraire. Ce dernier faisait allusion, en les désapprouvant, aux alliances conclues avec les barbares : il écrivit son commentaire en 407 , un an après l'incursion des Germains en Gaule et en Italie, jusqu'aux abords de Florence. Il faisait partie des barbarophobes, méfiants à l'égard du général Stilicon. La critique a porté puisque Stilicon a reproché ce commentaire à Jérôme ${ }^{12}$. Raban reprend l'expression "notre époque ». Cela procède du sentiment de vivre dans le même monde que celui de Jérôme, conformément à une conception augustinienne du temps : l'humanité est entrée, depuis l'Incarnation, dans son dernier âge ; les événements sont récurrents et parlent de la lutte entre la Cité de Dieu et celle du diable, lutte qui ne cessera qu'à la fin des temps. Raban est sans doute convaincu de l'actualité du commentaire de Jérôme, même s'il y voit des événements différents. Il écrit son commentaire au cours d'une période de disgrâce, entre 842 et 847 : il avait pris le parti de Lothaire, mais son abbaye, Fulda, se trouvait dans le royaume de Louis. La reprise de l'exégèse de Jérôme fait probablement écho, dans son esprit, à l'actualité des années 840, aux guerres civiles qui viennent de ravager l'Empire, menacé par diverses nations, les Normands et les Sarrasins. Raban dénonce ici, à travers les mots de Jérôme, la faiblesse de l'Empire et les mauvais compromis de ses gouvernants.

9 Haymon d'Auxerre, qui écrit entre 840 et 860, soit à partir de l'éclatement de l'Empire, va plus loin encore. Infléchissant l'explication de Jérôme à propos du verset de Dn 2, 43, c'est-à-dire de la statue aux pieds d'argile mêlée de fer, il souligne la dégénérescence en cours. Cette modification est remarquable dans une exégèse qui se veut traditionnelle. Selon Jérôme, les jambes de fer et les pieds de la statue figurent l'Empire romain. La dégradation des matériaux correspond à sa puissance décroissante. Le moine de Bethléem 
ne commente pas la nature du mélange des matériaux des pieds, alors qu'Haymon déclare :

2, 40. LE QUATRIÈME ROYAUME est celui des Romains qui d'abord vainquirent le monde presque entier, mais ensuite, on découvrit qu'eux non plus n'étaient pas invincibles, comme on le voit maintenant.

2, 43. ILS SERONT MÊLÉs, CERTES, PAR UNE SEMENCE HUMAINE, c'est-à-dire qu'ils ne seront pas en terre cuite et en argile mais des hommes, descendant de la race des prédécesseurs. MAIS ILS N'ADHÈRERONT PAS LES UNS AUX AUTRES, car ils seront en désaccord entre eux; ils n'auront pas autant de puissance que leurs ancêtres et seront dégénérés par rapport à ces derniers ${ }^{13}$.

10 Le texte en gras montre les idées introduites par Haymon d'Auxerre. L'idée de dégénérescence est renforcée par rapport à sa source, Jérôme. Haymon souligne la puissance moindre et le désaccord des héritiers. Il semble ainsi dénoncer les discordes entre les fils de Louis le Pieux, qui rompent l'unité de l'Empire ainsi que la paix et la concorde en son sein. Il est d'autant plus possible qu'Haymon fasse allusion au partage de l'Empire entre les fils de Louis le Pieux et aux luttes qui s'en sont suivies qu'Auxerre se trouve tout près de Fontenoy. C'est là qu'en 841 se déroula la bataille opposant Charles et Louis à leur frère âné Lothaire. La jonction des troupes de Charles et de Louis eut lieu à Auxerre $^{14}$. Le carnage fut tel qu'il assombrit la victoire des deux cadets et frappa l'esprit des contemporains ${ }^{15}$. Peu après, selon Heiric d'Auxerre (disciple d'Haymon), Charles s'est rendu à Saint-Germain pour assister à la première translation des reliques du patron de l'abbaye, à l'occasion du début des travaux décidés par Conrad I ${ }^{\mathrm{er}}$, oncle de Charles et abbé laïque du monastère ${ }^{16}$. Les moines de Saint-Germain ne pouvaient donc être indifférents à cet événement. Parmi les autres expositions carolingiennes sur Daniel, seule l'anonyme attribuée à Remi d'Auxerre ou Israël Scot se rapproche de l'interprétation d'Haymon, sans être aussi nette: «ILS SERONT MÊLÉS PAR UNE SEMENCE HUMAINE MAIS N'ADHÉRERONT PAS ENTRE EUX, c'est-à-dire : ils ne seront pas aussi forts que leurs ancêtres le furent ${ }^{17}$. " Comme chez Haymon, la dégradation est dynastique, alors que chez Jérôme elle concerne la puissance militaire globale de l'Empire, par rapport aux barbares ${ }^{18}$. Mais l'auteur insiste moins que le moine d'Auxerre sur la dégénérescence et n'évoque absolument pas la discorde.

11 Les Carolingiens sont donc particulièrement sensibles au thème de la succession des empires, qu'ils relient à l'affaiblissement de l'Empire, voire à la décadence de la dynastie carolingienne. Il ne faudrait cependant pas penser que le passage ne pouvait s'expliquer qu'en ce sens. À la fin du Moyen Âge, Jean Gerson voit dans la statue de Dn 2 une figure de l'État: le corps politique est souvent comparé, alors, à un corps humain ${ }^{19}$. L'État existe désormais en tant qu'entité abstraite, dépersonnalisée : l'interprétation du Livre est bien liée à une société donnée, à un mode de représentation précis.

12 Un autre exemple de ce modelage de l'exégèse au gré de la manière dont on conçoit le politique concerne la vision de l'arbre coupé, au chapitre 4 de Daniel. L'image renvoie au châtiment infligé à Nabuchodonosor, coupable d'orgueil - il était persuadé d'être responsable de sa très grande puissance: le souverain est évincé du trône durant une période de sept unités indéterminées. Les nombreuses références à ce récit permettent de saisir l'importance du discours sur la caducité du pouvoir terrestre à l'époque carolingienne.

13 L'épisode est généralement utilisé, dans le sillage de Jérôme, pour dénoncer l'orgueil qui éloigne les hommes de Dieu, tout spécialement les puissants que leur gloire humaine 
illusionne. Dhuoda, aristocrate septimanienne, met ainsi en garde son fils dans l'ouvrage qu'elle consacre à son éducation :

Notre manière de parler dans le siècle est ainsi faite que nous soumettons à notre propriété n'importe quel objet, alors qu'il n'en est pas ainsi. Quelqu'un, dans le siècle, revendique en disant : 'C'est mon royaume' et : 'dans tout mon royaume', et il ne réfléchit pas qu'au Seigneur appartiennent le royaume et tous ceux qui s'y meuvent. Nabuchodonosor, par exemple, homme sans foi ni loi, vaincu et terrassé, et comme converti, déclare : il est le roi qui commande et dirige, qui a le pouvoir d'élever. Ceux qui marchent dans l'orgueil, il peut les abaisser. Il possède le royaume et il donnera à qui il veut ${ }^{20}$.

14 Les miroirs aux princes carolingiens regorgent d'allusions à cet épisode. Jonas d'Orléans y fait référence afin de montrer que toute royauté est attribuée aux justes et par Dieu seul. Au chapitre 6, l'évêque d'Orléans déclare :

De fait, le royaume s'effondre par l'injustice. [...] Et Daniel dit à Balthasar : Ô roi ! Le Dieu très haut a accordé la royauté, la grandeur, la gloire et la majesté à Nabuchodonosor ton père; et à cause de la grandeur qu'il lui avait accordée, tous les peuples, nations et langues tremblaient de crainte en sa présence ;il tuait qui il voulait et frappait qui il voulait. Il élevait qui il voulait et abaissait qui il voulait. Et lorsque son cour s'éleva et que son esprit s'endurcit jusqu'à l'arrogance, il fut déposé de son trône royal et on lui retira sa gloire. Il fut chassé d'entre les hommes et son coeur devint semblable à celui d'une bête. [...] Ces paroles montrent clairement que la piété, la justice et la miséricorde consolident la royauté ${ }^{21}$.

Dans le chapitre suivant, Jonas dit: « La royauté terrestre n'est pas donc pas donnée par les hommes mais par Dieu; c'est ce qu'atteste le prophète Daniel: Par la sentence des Vigilants ont été décrétés la parole des saints et leur requête, afin que les vivants sachent que le Très Haut a le pouvoir sur la royauté des hommes, qu'il la donnera à qui il veut et y élèvera le plus humble des hommes ${ }^{22}$ ». De même, Hincmar de Reims cite Dn 4, 26-29 en exemple de châtiment pour la superbe des tyrans, tandis que Sedulius Scot évoque l'épisode dans le Liber de rectoribus christianis composé vers 855-859 à l'adresse, pense-t-on, de Lothaire II $^{23}$.

Cette sensibilité à la caducité du pouvoir terrestre, soumis à l'approbation divine et à l'humilité de son détenteur, est également soulignée dans l'exégèse d'Haymon d'Auxerre ${ }^{24}$ . Ce dernier infléchit à nouveau le commentaire de Jérôme - comme l'indiquent les caractères en gras dans le texte suivant - afin d'insister sur l'exigence morale pesant sur le détenteur d'une fonction politique :
4, 12. 20. LA SOUCHE DE SES RACINES, LAISSEZ-LA DANS LA TERRE. Il montre que le roi doit être déposé mais que son royaume lui demeurera. ET QU'IL SOIT ATTACHÉ AVEC UNE CHAÎNE DE FER ET D'AIRAIN, c'est-à-dire très durement éprouvé, ou du moins qu'il soit attaché comme un fou afin qu'il ne se jette pas < sur d'autres > [...].
4, 14. SELON L'AVIS DES VEILLEURS, c'est-à-dire la décision des anges. EN EFFET, IL Y EUT UNE PAROLE ET UNE REQUÊTE de ces derniers dont il opprimait les peuples pour qu'il fût déposé $[\ldots .].]^{25}$.

Il est difficile de ne pas mettre en relation cette avalanche de références au châtiment public du roi orgueilleux ou inique avec la réflexion théorique qui accompagne la déposition effective de Louis le Pieux, en 833. Les évêques qui accomplirent l'acte souhaitaient qu'il fût définitif, mais ils étaient conscients que cela était insuffisant puisqu'ils tentèrent, en vain, de pousser Louis à un véritable retrait du monde, par la conversion monastique ${ }^{26}$. L'empereur semble avoir accepté - et non désiré - la déposition, sachant qu'elle serait temporaire ${ }^{27}$. Miroirs des rois et exégèse bibliques livrent sur le sujet une réflexion théorique en faveur de la déposition. 

l'ouverture du champ herméneutique et donc le sens qui est privilégié par les Carolingiens. Selon la Bible, l'arbre haut, feuillu et porteur d'un fruit abondant, est une représentation du roi. Pour Haymon, ces caractères évoquent l'excellence et la richesse du royaume, signes de la puissance personnelle du roi. Au XIII e siècle en revanche, Albert le Grand prolonge l'explication : les branches de l'arbre sont « les princes et les préfets », ses feuilles $"$ ses édits et ses ordres $»^{28}$. L'arbre représente désormais le royaume: le développement des théories politiques conduit à se représenter l'État comme un tout cohérent. Le politique est moins personnalisé, et de ce fait moins lié à un discours moral. Pour autant, les visions politiques de Daniel donnent lieu, dès le IX ${ }^{e}$ siècle, à une réflexion sur l'équilibre des pouvoirs.

19 C'est parce qu'il sait décrypter les songes des rois que Daniel est un conseiller de ces derniers. Sa sagesse surpasse celle des mages, magiciens et devins ; bien qu'il soit juif, les rois babyloniens et perses le consultent (Dn 2, Dn 5). Il est donc considéré, à l'époque carolingienne, comme un modèle de conseiller chrétien pour les rois. Dhuoda exhorte plusieurs fois son fils à suivre cet exemple :

Encore enfants, mais dans toute la fleur de leur jeunesse, Samuel et Daniel ont jugé des vieillards comme faisaient leurs pères; ils furent dans le monde les puissants conseillers des rois et même des chefs païens d'une nation étrangère : les conseils toujours loyaux qu'ils donnèrent leur valurent d'obtenir la digne récompense de leurs mérites et la palme de la victoire. Considère les anciens Pères, considère Joseph devant Pharaon, Daniel devant Nabuchodonosor, Balthasar, Darius et les chefs des Perses ou des Mèdes: sans rien renier de leurs convictions, ils furent toujours pour eux les meilleurs conseillers ${ }^{29}$.

Cet exemple évoque les deux qualités politiques de Daniel. D’une part, il est de jugement sûr car il est l'homme de Dieu; d'autre part, il met son esprit averti au service de rois divers, y compris des païens : il intervient dans le « monde ». C'est à ce même modèle que recourt Raban Maur. Dans la lettre de dédicace accompagnant son Commentaire sur Daniel et destinée au roi dont il dépend, Louis le Germanique, Raban utilise les deux facettes politiques de Daniel. D'une part, en tant qu'homme impliqué dans les affaires de son temps, Daniel constitue un modèle pour le roi chrétien:

En effet, j'ai pensé qu'il convenait que je t'envoie les actions très estimées et les révélations mystiques consignées dans le livre de ce prophète, dont ni le tourment d'une persécution hostile, ni la puissance terrestre, ni la félicité temporelle n'ont changé la perfection mais l'ont éprouvée, à toi qui, établi sur le trône, a appris à ne pas te décourager des malheurs du monde et à ne pas t'élever dans l'orgueil en raison des bonheurs de ce siècle, mais qui te hâtes, en t'avançant par la voie royale, de parvenir au royaume éternel et vers la récompense de la vie éternelle, afin que, t'appliquant à leur méditation avec zèle, tu imites l'ardeur de celui qui s'est acquitté des affaires du monde sans renoncer à rien concernant le service de Dieu et afin que tu sois, en même temps que le prophète, appelé avec raison, selon le mot de l'ange, homme de désirs ${ }^{30}$, non pas charnels, mais spirituels, non pas terrestres, mais célestes 31 .

21 On saisit bien, dans cet extrait, l'exigence morale qui légitime, aux yeux de Raban, l'exercice du ministère royal. Implicitement cependant, l'exégète qui éclaire son souverain par une explication biblique se place lui-même dans la position du conseiller, d'un homme au savoir supérieur puisque inspiré par Dieu. C'est d'ailleurs un rôle de médiation que Raban revendique un peu plus loin dans sa dédicace: «Puisses-tu donc apprendre que ma petitesse implore par des prières dévouées et avec zèle, afin que tu 
tiennes avec bonheur le royaume terrestre sous le gouvernement divin, pendant le temps de ta $v^{32}{ }^{32}$.» Dans le commentaire original qu'il livre à propos de l'épisode deutérocanonique de Bel (Dn 14), Raban évoque à nouveau ce couple roi-prophète, qui répond à l'idéologie des deux ministères développée dans l'entourage de Louis le Pieux ${ }^{33}$. Son explication est une véritable illustration du principe politique de coopération entre les religieux médiateurs de la divinité, détenteurs de l'auctoritas des pontifes, et le roi, qui doit mettre à leur service sa potestas. Après avoir découvert, grâce à Daniel, la supercherie des prêtres de l'idole de Bel, le roi rejette ce dernier :

Le roi livra Bel au pouvoir de Daniel, qui le détruisit, ainsi que son temple, quand Jésus-Christ, qui est le roi des rois et le seigneur des seigneurs, et qui, étant victorieux de ses adversaires et humiliant la puissance terrestre, soumit la terre à ses prédicateurs; ces derniers, détruisant l'idolâtrie et renversant les temples des démons, affermirent en tous lieux le culte du seul vrai Dieu en enseignant la parole du seigneur et en purifiant, par le sacrement du baptême, les hommes des péchés ; et ils firent des rois du monde les serviteurs de Dieu, les constructeurs des églises du Christ et leurs aides dans la prédication de l'Évangile, de telle sorte que ces derniers ordonnaient, dans leur royaume, de détruire les images des idoles, de fermer leurs temples, d'ouvrir les églises sacrées et d'offrir aux fidèles la possibilité d'y entrer. On lit dans les histoires que les empereurs Constantin et Théodose agirent de la sorte, ainsi que d'autres rois chrétiens en grand nombre ${ }^{34}$.

La nécessaire synergie entre les détenteurs des ministères fait probablement écho à la situation personnelle de Raban lorsqu'il compose ce commentaire ; c'est sans doute aussi une manière de rappeler à Louis qu'il a besoin de conseillers ecclésiastiques chevronnés, tel que Raban lui-même.

Le livre de Daniel alimente donc les discours politiques carolingiens qui transparaissent non seulement dans l'exégèse contemporaine mais aussi dans les références à certains de ses épisodes : ces derniers légitiment un précepte moral dans divers miroirs ou servent de matrice à des visions renouvelées, à propos d'événements récents. Deux épisodes ont été privilégiés pour leur dimension politique : la vision de la statue aux pieds d'argile, qui évoque la décadence de la dynastie à partir des années 840 , et celle de l'arbre coupé, emblématique de la caducité du pouvoir terrestre. Le personnage de Daniel lui-même, saint au service des princes, a servi de modèle pour évoquer les qualités d'un bon conseiller royal, qu'il s'agisse d'un aristocrate laïc ou du détenteur de l'autorité et de l'admonition. Ce dernier aspect fait référence au cadre ministériel de la coopération entre les prélats-exégètes dotés d'un savoir exceptionnel - à l'image du prophète - et les détenteurs de la puissance terrestre, en particulier les rois. Daniel a cependant nourri d'autres visions et représentations politiques, dans la suite du Moyen Âge: l'interprétation de la Bible, polymorphe, s'adapte indéfiniment aux sociétés qui la lisent.

\section{NOTES}

1. Voir M. DULAEY, Des forêts de symboles. L'initiation chrétienne et la Bible (I $I^{e r}-I V^{e}$ siècles), Paris, 2001, p. 157-189. 
2. Le texte de Pierre de Pise est édité dans PL 96 (1347B-1362D) ; voir aussi M. GORMAN, « Peter of Pisa and the Quaestiunculae copied for Charlemagne in Brussels II 2572 », Revue bénédictine, 110, 2000, p. 238-260, qui traite justement du manuscrit donnant ce texte de Pierre, parmi d'autres (Bruxelles, Bibliothèque Royale II 2572, f.1v-16v pour le traité sur Daniel). Le deuxième commentaire carolingien, la glose de Saint-Gall, est transmis par Sankt-Gallen Stiftsbibliothek 41 et a fait l'objet de deux études : S. BERGER, Histoire de la Vulgate pendant les premiers siècles du Moyen Âge, Nancy, 1893, p. 135-136 et J. DE BLIC, "L'œuvre exégétique de Walafrid Strabon et la Glossa ordinaria », Recherches de théologie ancienne et médiévale, 16, 1949, p. 5-28. Le commentaire de Raban Maur, inédit, est transmis de manière lacunaire par un seul témoin, Karlsruhe, Landesbibliothek Aug. Perg. 208, p. 2-83 et ses sources ont été identifiées par S. CANTELLI-BERARDUCCI, Hrabani Mauri Opera Exegetica. Repertorium fontium, 3 vol., Turnhout, 2007, spéc. vol. 2. Nous avons établi l'édition critique de l'Annotation brève sur Daniel d'Haymon d'Auxerre dans notre thèse de doctorat (Exégèse et politique dans l'œuvre d'Haymon d'Auxerre, thèse soutenue en 2006 à l'Université de Paris Sorbonne-Paris IV) à paraître dans la collection Corpus christianorum continuation medieualis, à partir des trois témoins subsistant (Albi, B. M. 31, 61r-72v, Barcelona, Catedral 64, 117rb-124rb et Torino, B.N. 531 D. V. 17, 241v-252v). Enfin, le commentaire de l'anonyme qui serait $\mathrm{du}_{\mathrm{IX}} \mathrm{e}^{\mathrm{ou}} \mathrm{du} \mathrm{X}^{\mathrm{e}}$ siècle est transmis par Munich, Bayerische Staatsbibliothek clm 3704 (f. 166v-170v) et Paris, B.N.F, n.a.l. 762 (f. 124v-128) ; les hypothèses d'attribution sont variées mais aucune d'entre elles ne se fonde sur une critique publiée : il pourrait s'agir de Wigbod ou de Remi d'Auxerre selon F. Stegmüller ( Repertorium Biblicum Medii Aevi, Madrid, V. Commentaria. Auctores R-Z (1957), n. 7220 pour Remi, n. 8377 pour Wigbod et IX. Supplementi Altera Pars. Glossa ordinaria, 1977) ou encore d'Israël Scot selon Burton Edwards, qui nous a fait part de cette hypothèse de façon informelle. Sur Israël Scot, voir C. JEUDY, « Israël le Grammairien » dans le Dictionnaire d'histoire et de géographie ecclésiastique, 26, fasc. 151, Paris, 1997, c. 311-313. Précisons que deux autres œuvres carolingiennes ont peut-être existé, sans qu'il en reste de trace. Angélome de Luxeuil a peut-être commenté Daniel (F. STEGMÜLLER, Repertorium..., II, 1950, n. 1339, 1). M. Zier (« The Medieval Latin Interpretation of Daniel : Antecedents to Andrew of Saint-Victor », Recherches de théologie ancienne et médiévale, 58, 1991, p. 43-78, spéc. p. 58, n. 42) suggère l'existence d'une compilation carolingienne d'extraits de Grégoire le Grand, qui aurait pu servir de source à celle du XII ${ }^{\mathrm{e}}$ siècle, éditée sour le nom de Paterius (PL 79, 997A-1002D : De testimoniis in Danielem prophetam); à ce sujet, voir A. WILMART, « Le recueil grégorien de Paterius et les fragments wisigothiques de Paris ", Revue bénédictine, 39, 1927, p. 81-104 (p. 94-101 sur Daniel).

3. L'œuvre d'Haymon d'Auxerre est éditée : In Osee, éd. PL 117 (11A-98C) ; In Ioel, Amos, éd. sous le nom de Remi d'Auxerre par Marguerin de la Bigne, dans Maxima Bibliotheca Veterum Patrum, Lyon, 1677, vol. 16 (962G-294D) ; In Abdiam, Ionam, Micheam, Nahum, Habacuc, Sophoniam, Aggeum, Zachariam, Malachiam, éd. PL 117 (119D-294D). Le manuscrit Sankt-Gallen Stiftsbibliothek 41 contient aussi une glose sur Osée et une sur Zacharie (p. 229-295). Enfin, les commentaires d'Otfrid sont inédits.

4. On ne connaît pas de manière sûre les dates de rédaction de la Glose de Saint-Gall et du commentaire anonyme, qu'on suppose être des $\mathrm{IX}^{\mathrm{e}}-\mathrm{X}^{\mathrm{e}}$ siècles ; voir à ce sujet la bibliographie indiquée ci-dessus en n. 2 ; l'inventaire des manuscrits du IX ${ }^{\mathrm{e}}$ siècle effectué par B. Bischoff (Katalog der festländischen Handschriften des neunten Jahrhunderts (mit Ausnahme der wisigotischen), I, Aachen-Lambach, Wiesbaden, 1998 ; II, Laon-Paderborn, 
Wiesbaden, 2004) n'étant publié que de façon partielle, nous ignorons ce que Bernhard Bischoff pensait de la datation du manuscrit de St-Gall.

5. Y.M.-J. CONGAR, L'Ecclésiologie du haut Moyen Âge de saint Grégoire le Grand à la désunion entre Byzance et Rome, Paris, 1968, p. 273.

6. FRÉCULPHE DE LISIEUX, Opera omnia, éd. M.I. ALLEN, CCCM 169 et 169 A, Turnhout, 2002, respectivement I, 3, 16 et I, 3, 18 .

7. Pour Flavius Josèphe, voir De Antiquitatibus Iudaeorum Ruffino presbytero interprete, Basileae, apud I. Frobenium, 1524 ; pour Orose, voir Histoires contre les païens, éd. et trad. M.-P. ARNAUD-LINDET, Paris, 3 vol. 1990-1991.

8. FRÉCULPHE DE LISIEUX, Opera omnia, op. cit., I, 7, 13 ; II, 1, 3 ; II, 1, 23.

9. P. E. DUTTON, The Politics of Dreaming in the Carolingian Empire, University of Nebraska, 1994, p. 200-210. Pour l'édition, voir Ph. JAFFÉ, Visio Caroli magni, dans ID., Monumenta carolina, Berlin, 1867, p. 701-704.

10. NotKer, Gesta Karoli Magni, 1, 1, éd. H. F. HAEFELE, MGH Script. rerum germ. 12, cité par P. E. DUTTON, The Politics of Dreaming..., op. cit., p. 199-200.

11. RABAN MAUR, Commentaire sur Daniel, op. cit. n. 2, f. 14v-15r : « [...] sed pedes eius et digiti ex parte ferrei, ex parte fictiles sunt, quod hoc tempore manifestissime comprobatur : sicut enim in principio nihil romano imperio fortius et durius fuit, ita in fine rerum nihil imbecillius, quando et in bellis ciuilibus et aduersum diuersas nationes aliarum gentium barbararum indigemus auxilio. » Nous empruntons la traduction de Jérôme à R. COURTRAY, Le Commentaire sur Daniel de Jérôme, traduction, notes et commentaire, édition critique du De Antichristo, thèse de doctorat soutenue à l'université de Lyon II en 2004, 2 vol. (spéc. vol. 2, p. 373-374).

12. Ibid., n. 165 , p. 374.

13. Sur Dn 2, 43-43 : «REGNVM QVARTVM regnum est Romanorum qui primo pene uniuersum mundum domuerunt, sed postmodum ipsi domabiles inuenti sunt, sicut modo cernitur. COMMISCEBVNTVR QVIDEM HVMANO SEMINI, id est non erunt testa et lutum, sed homines de genere priorum descendentes. SED NON ADHEREBVNT SIBI, quia discordantes erunt inter se ; sed neque tantĘ uirtutis erunt quantĘ priores eorum fuerunt, eruntque degeneres ab illis. "

14. J.L. NELSON, Charles le Chauve, 1992, trad. française D.-A. CANAL, Paris, 1994, p. 139. 15. Ibid., p. 141 ; G. BÜHRER-THIERRY, L'Europe carolingienne (714-888), Paris, 2004, p. 54. Voir par exemple les Annales lyonnaises : « 841. Et lacrimabile bellum inter filios imperatoris Hludowici haut procul ab urbe Autisiodorensium, in quo christianus utrimque populus mutua se caede prostrauit 7 Kal. Iul. » (Annales Lugdunsenses, MGH SS, t. 1, éd. G.H. PERTZ, Hanovre, 1826, p. 110).

16. HEIRIC D'AUXERRE, Miracula sancti Germani, éd. L.-M. DURU, Bibliothèque historique de l'Yonne, II, Auxerre, 1863, p. 114-182 (2, 2, 96-97, p. 164-165).

17. ANONYME, Commentaire sur Daniel 2,43 : « COMMISCEBVNTVR HVMANO SEMINE SED NON ADHEREBVNT SIBI, id est non erunt ita fortes sicut priores illorum fuerunt. » Voir Munich, Bayerische Staatsbibliothek, clm 3704, f. 166v-170v (spéc. f. 167v) et Paris, B.N.F., n.a.l. 762, 125v-128r (spéc. f. 126v).

18. Ce lieu est d'ailleurs celui où les deux commentaires sont les plus proches; il nous paraît cependant insuffisant pour parler d'une dépendance d'un texte vis-à-vis de l'autre. 19. Voir le discours Rex in sempiternum uiue cité par J.-P. BOUDET, « Le bel automne de la culture médiévale ( $\mathrm{XIV}^{\mathrm{e}}-\mathrm{XV}^{\mathrm{e}}$ siècle) », dans Histoire culturelle de la France. Le Moyen Âge, Paris, 1997, p. 294. 
20. DHUODA, Manuel pour mon fils, éd., introduction, et notes de P. RICHÉ, trad. B. DE VREGILLE et CL. MONDÉSERT, Paris, 1975 [2éd. augmentée 1997], SC 225bis, I, 5 : « Vsus locutionis nostrae in saeculo talis est, ut cuiuslibet rem in nostram uetamur potestatem, cum non ait ita. Contendit quia in saeculo et dicit : 'Meum est regnum', et 'in toto meo regno', et non considerat quia domini est regnum et omnium currentium in eo. Nam et Nabugodonosor incredulus pessimusque, uictus et prostratus et quasi recuperatus, ita afatus est dicens : 'Ipse est rex imperans et regens, habens potestatem exaltandi, et eos qui in superbia gradiuntur potest humiliare, habensque regnum et cui uoluerit dabit illud'. »

21. JONAS D’ORLÉANS, Le Métier de roi, éd., trad. et notes A. DUBREUCQ, SC 407, Paris, 1995, chapitre 6 : «Quod uero per iniustitiam cadat [...] Danihel loquitur ad Balthasar : 'O rex, deus altissimus regnum et magnificentiam et gloriam et honorem dedit Nabuchodonosor patri tuo et propter magnificentiam, quam dederat ei, uniuersi populi, tribus et linguae tremebant et metuebant eum ; quos uolebat interficiebat et quos uolebat percuciebat; quos uolebat exaltabat et quos uolebat humiliabat. Quando autem eleuatum est cor eius et spiritus illius obfirmatus est in superbiam, depositus est de solio regni sui et gloria eius ablata est et a filiis hominum eiectus est, sed et cor eius cum bestiis positum est' [...] Quibus uerbis liquido claret, quod pietas, iustitia et misericordia stabiliant regnum. » 22. «Quod autem non ab hominibus, sed a deo regnum terrenum tribuatur, Daniel propheta testatur dicens : 'In sententia uigilum decretum est et sermo sanctorum et petitio, donec cognoscant uiuentes, quod dominetur excelsus in regno hominum et cuicumque uoluerit dabit illud et humillimum hominem constituet super illud'. » Jonas fait également référence à l'épisode du festin de Balthasar (Dn 5) à l'appui de sa démonstration.

23. HINCMAR, De cauendis uitiis et uirtutibus exercendis, I, 3 éd. D. NACHTMAN, Munich, 1998 (MGH Quellen... 16). Pour Sedulius Scot (Liber de rectoribus christianis, 3, éd. S. HELLMANN dans Quellen und Untersuchungen zur lateinischen Philologie des Mittelalters I, 1, Munich, 1906, p. 1-91 [spéc. p. 29]), le fait est signalé par H. H. ANTON, Fürstenspiegel und Herrscherethos in der Karolingerzeit, Bonn, 1968, p. 267.

24. On ne conserve malheureusement pas le commentaire de Raban Maur sur ce passage.

25. HAYMON D'AUXERRE, Annotation brève sur Daniel 4, 12. 20 : « GERMEN RADICVM EIVS IN TERRA SINITE. Ostendit deponendum regem, set regnum eius ipsi mansurum. ET ALLIGETVR VINCVLO FERREO ET ĘREO, id est durissima temptatione, uel certe quasi insanus uinciatur ne se precipitet $[. .] 4,$.14 . IN SENTENTIA VIGILVM, id est angelorum decreto. Fuit enim SERMO ET PETITIO eorum ut deponeretur, quorum gentes oprimebat. »

26. M. DE JONG, «Sacrum palatium et ecclesia. L'autorité religieuse royale sous les Carolingiens (790-840) », Annales. Histoire, Sciences sociales, 2003/6, p. 1243-1269 (p. 1267-1268).

27. Ibid., p. 1266-1268.

28. ALBERT LE GRAND, Commentarius in Danielem, dans ID., Opera omnia, A. Borgnet éd., Paris, 1890-1899, XVIII, p. 447-642, spéc. IV, 15 : « ET RAMI EIVS PVLCHERRIMI, principes scilicet et praefecti. Ibid., IV, 10 : ET EXCVTITE FOLIA EIVS. Folia sunt uerba in edictis et mandatis, quae uirtute erant plena, cum erant in potestate. »

29. DHUODA, Manuel pour mon fils, op. cit. n. 20, III, 5 : «Certe Samuhel et Danihel pueri, in iuuentutis flore uigentes, more patrum senes iudicauerunt, et consiliarii magni regibus in saeculo fuerunt, etiam et ducum gentilium externaeque gentis, fidele dantes consilium, merita accipere digna promueruerunt uictoris palmam. Item. Considera patres priores, considera Ioseph erga Pharaonem, Danielem erga Nabugodonosor, Baltasar, Dariumque et 
ducibus Persarum atque Medorum : sua non deserentes utilissimi illi ad consilium fuerunt semper. »

30. Dn 10, 19.

31. MGH, Epist. 5, Karolini Aeui 3, éd. E. DÜMMLER, Weimar, 1899, n. 34, p. 468 sq. : « Dignum enim arbitratus sum, ut illius prophetae, cuius perfectionem nec tribulatio hostilis persecutionis nec potestas terrena et felicitas temporalis mutauit, sed probauit, tibi, qui in regno constitutus nec aduersitatibus mundanis frangi nec prosperitatibus huius SĘculi in superbiam erigi nosti, sed uiam regiam incedendo ad regnum perpetuum et premium aeternae uitae peruenire festinas, actus probatissimos et reuelationes misticas in libro conscriptas dirigerem, ut eorum meditatione sedulo incumbens eius studium imiteris, qui sic impleuit negotium mundanum, quod nequaquam aliquod omitteret ad officium pertinens diuinum appellerisque rite simul cum propheta angelico affatu uir desideriorum, non utique carnalium, sed spiritalium, non terrenorum, sed caelestium. Alioquin uiri uocabulum non rite teneres, si mollitiem uoluptatum huius s̨̨culi sequereris, et non magis desiderium regni caelestis. »

32. Ibid. : « Hoc ergo meam paruitatem deuotis precibus sedulo orare cognoscas, ut regnum terrenum sub diuina gubernatione tempore uitae tuae feliciter teneas. " 33. À propos de ces ministères, autorité pontificale et puissance royale, voir par exemple Y. SASSIER, Royauté et idéologie au Moyen Âge. Bas Empire, monde franc, France (IVe-XII siècle), Paris, 2002, p. 136-144.

34. Commentaire sur Daniel 14, 19-21 (ms Karlsruhe, Landesbibl. Aug. perg. 208, f. 68r) : « rex et tradidit Bel in potestatem Danielis qui subuertit eum et templum eius, cum Ihesus Christus qui est rex regum et dominus dominorum, aduersarios suos superans, et terrenam potentiam humilians, subdidit orbem predicatoribus suis qui, destruentes idolatriam et subuertentes templa demoniorum, culturam unius ueri dei uerbum domini docendo et baptismatis sacramento homines a peccatis abluendo ubique roborabant ipsosque reges mundi fecerunt esse seruos dei et constructores aecclesiarum Christi, adiutoresque predicationis euangelii, ita ut iuberent ubique in regno suo destruere idolorum simulacra, et templa eorum claudere, aeclesiasque sacras aperire et fidelibus illuc facultatem intrandi praebere, sicut legimus in historiis Constantinum et Theodosium imperatores fecisse et alios christianos reges quam plurimos. »

\section{RÉSUMÉS}

L'époque carolingienne voit le renouveau de l'exégèse en général et de celle de Daniel en particulier. Le thème de la succession des empires, celui - qui lui est lié - de la caducité du pouvoir terrestre, ont rencontré un certain écho après l'éclatement de l'Empire, en 843. De même, la figure de ce prophète, médiateur choisi par Dieu et conseiller des rois, a servi de modèle à une époque durant laquelle le pouvoir est théorisé en termes de ministères complémentaires et dans la pratique duquel les liens personnels sont importants. On voit ainsi comment les carolingiens, exégètes, auteurs de miroirs ou rédacteurs de visions, ont utilisé la matrice biblique et patristique pour donner un sens à l'histoire qu'ils vivaient et légitimer certains actes politiques. 
Daniel and Political Visions in the Carolingian Era. During the Carolingian era, biblical exegesis, especially on the book of Daniel, underwent a revival. The topic of the succession of empires, the one - which is linked - of the deciduous nature of earthly power, met the concerns of the Carolingians after the break-up of the Empire in 843. The figure of the Prophet, a mediator chosen by God and an adviser of kings, was used as a model : during the period, the power was theorized in terms of completing ministeria and was practiced according to important personal relationships. We can appreciate thus how the Carolingian scholars - biblical commentators, authors of specula or uisiones - used the biblical and patristic matrix in order to give a meaning to the historical events of their time, and to legitimate some political actions.

\section{INDEX}

Mots-clés : exégèse, miroirs, prophète, visions

Keywords : exegesis, mirrors, prophet

\section{AUTEUR}

\section{SUMI SHIMAHARA}

Université de Paris IV-Sorbonne, UFR d'histoire, 1 rue Victor Cousin, F-75230 Paris cedex 05 